\title{
Treatment by specialist surgical neurooncologists improves survival times for patients with malignant glioma
}

\author{
Ursalan A. Khan, MB, ChB, MRes, ${ }^{1}$ Amar Bhavsar, MBBS, BSc, ${ }^{1}$ Hasan Asif, ${ }^{2}$ \\ Konstantina Karabatsou, FRCS(SN), ${ }^{1}$ James R. S. Leggate, FRCS, ${ }^{1}$ Ajit Sofat, FRCS(SN), ${ }^{1}$ \\ and lan D. Kamaly-AsI, MD, MBChB, FRCS(SN) ${ }^{1}$ \\ 1Department of Neurosurgery, Greater Manchester Neurosciences Centre, Salford Royal Hospital, Salford; and ${ }^{2}$ Faculty of \\ Medicine, Imperial College London, London, United Kingdom
}

\begin{abstract}
OBJECT Surgeries for CNS tumors are frequently performed by general neurosurgeons and by those who specialize in surgical neurooncology. Subspecialization in neurosurgical practice has become common and may improve patient morbidity and mortality rates. However, the potential benefits for patients of having their surgeries performed by surgical neurooncologists remain unclear. Recently, a shift in patient care to those who practice predominantly surgical neurooncology has been promoted. Evidence for this practice is lacking and therefore requires fundamental investigation.

METHODS The authors conducted a case-control study of neurooncology patients who underwent surgery for glioblastoma and anaplastic astrocytoma during 2006-2009. Outcomes were compared for patients whose surgery was performed by general neurosurgeons (generalists) or by specialist neurooncology neurosurgeons (specialists). An electronic record database and a picture archiving and communication system were used to collect data and assess the extent of tumor resection. Mortality rates and survival times were compared. Patient comorbidity and postoperative morbidity were assessed by using the Waterlow, patient handling, and falls risk assessment scores. Effects of case mix were adjusted for by using Cox regression and a hazards model.

RESULTS Outcomes for 135 patients ( 65 treated by generalists and 70 by specialists) were analyzed. Survival times were longer for patients whose surgery was performed by specialists $(p=0.026)$ and after correction for case mix $(p=$ 0.019). Extent of tumor resection was greater when performed by specialists $(p=0.005)$ and correlated with increased survival times $(p=0.004)$. There was a trend toward reduced surgical deaths when surgery was performed by specialists $(2.8 \%)$ versus generalists $(7 \%)(p=0.102)$, and inpatient stays were significantly shorter when surgery was performed by specialists ( $p=0.008)$.
\end{abstract}

CONCLUSIONS The prognosis for glioblastoma multiforme remains dire, and improved treatments are urgently needed. This study provides evidence for a survival benefit when surgery is performed by specialist neurooncology neurosurgeons. The benefit might be attributable to increased tumor resection. Furthermore, specialist neurooncology surgical care may reduce the number of surgical patient deaths and length of inpatient stay. These findings support the recommendations for subspecialization within surgical neurooncology and advocate for care of these patients by specialists.

http://thejns.org/doi/abs/10.3171/2014.10.JNS132057

KEY WORDS glioma; glioblastoma multiforme; subspecialization; neurooncology; oncology

$\mathrm{D}$ ESPITE advances in surgical and medical treatments, brain tumors continue to be associated with a dismal prognosis, specifically for glioblastoma multiforme (GBM), for which the short median survival time is only 15 months at best. ${ }^{20}$ Consequently, there has been emphasis on subspecialization within surgical neurooncology and a drive to promote the care of these patients by specialists who practice predominantly surgical neurooncology. Many centers operate in this way, such that certain neurosurgeons are responsible for the treatment and care of these patients.

A recent development in the management of these cases has been a topic of debate in the United Kingdom. The National Institute of Health and Clinical Excellence, an or- 
ganization responsible for promoting best patient care, developed the Improving Outcomes Guidance for brain and CNS tumors. Its aims are to improve clinical outcomes and the quality of care provided. The Guidance states that neurosurgeons caring for tumor patients should devote at least $50 \%$ of their clinical activities to neurooncology. Implementation of this statement would promote subspecialization within surgical neurooncology and restrict the care of brain tumor patients to these surgeons. Although evidence exists for improved outcomes for patients under the care of subspecialists in other areas of neurosurgery, ${ }^{1,6,7}$ it remains unclear whether the same is true within surgical neurooncology. No recent study has analyzed the potential advantages, and a study published in 1998 showed no benefit, ${ }^{13}$

Traditionally, the management of malignant brain tumors has been seen as a core skill, and these cases have therefore been managed by most neurosurgeons. However, specialists would generally make more frequent use of surgical adjuncts including neuronavigation, brain mapping, intraoperative ultrasound, PET, functional MRI, framed and frameless stereotactic biopsies, and many more, which can influence patient outcomes. Some of these adjuncts are widely used by both general and specialist neurosurgeons; however, others might be relevant to specialists only. Furthermore, specialist surgical training allows for a greater experience in neurooncology, which could also contribute to possible differences in outcome. It is believed that specialists are more aggressive at removing tumor mass, but whether doing so is of benefit is still debatable. ${ }^{17}$ Despite these differences, fundamental investigations into differences in patient outcome when treatment is provided by a specialist surgical neurooncologist are still lacking, and therefore the advantages of subspecialization in this area remain unclear.

In consideration of this uncertainty, we designed this study to assess the potential differences in morbidity, surgical deaths, and survival times among patients for whom surgery was performed by a specialist surgical neurooncologist (hereafter referred to as a specialist) or a general neurosurgeon (hereafter referred to as a generalist). Because this study was a nonrandomized treatment comparison, we adjusted for case mix to reduce bias and improve the accuracy of our findings.

\section{Methods}

An electronic record database was used to identify all patients who had undergone surgery for a malignant supratentorial glioma during 2006-2009 at the Department of Neurosurgery in the Greater Manchester Neurosciences Centre, Salford Royal Hospital, Salford, United Kingdom. The malignant glial tumors included in the analysis were either GBMs or anaplastic astrocytomas (AAs). Patients were divided into 2 groups according to whether their surgery was performed by generalists or specialists. Because cases were treated by the surgeon on call at the time of referral, there was no case selection. Referrals were made from the Greater Manchester and Lancashire catchment area, and the on-call surgeon managed the cases. After patient referral, all cases were discussed at multidisciplinary meetings and otherwise received standard oncological management. Specialists were defined as the 4 core members of the neurooncology multidisciplinary team, all of whom devote at least $50 \%$ of their clinical activities to neurooncology.

In accordance with National Institute of Health and Clinical Excellence guidelines, the CNS tumor multidisciplinary team based at the Greater Manchester Neurosciences Centre discusses, without delay, every patient for whom imaging findings suggest a CNS tumor. Neuropathology and neuroradiology services are used and are involved in preoperative and postoperative management decisions and intraoperative diagnoses. Surgical approaches and intraoperative histological evaluations are also discussed. Palliative care specialists and allied health team members are included in the decision-making process.

Demographic information retrieved from the database included patient sex and age, presence and duration of preoperative seizures, and performance status before surgery, according to the WHO scale. Information about the type of surgery (biopsy or debulking) and extent of tumor resection (partial or gross macroscopic, identified by using postoperative imaging and radiology reports) was also collected. The Medical Research Council (MRC) prognostic index was calculated for each patient by using the extent of resection, patient age, history of seizures, and performance status to correct for case mix effects. ${ }^{19}$

Survival times and 30-day mortality rates were compared between groups. Differences in duration of inpatient stay and days from referral to multidisciplinary team meetings, from multidisciplinary team meetings to surgery, and from surgery to discharge were also analyzed. Postoperative complications were noted and compared. The benefits of neuronavigation on survival times and on extent of resection were analyzed by comparing surgeries that used neuronavigation with those that did not. Extent of resection was determined by postoperative imaging or radiotherapy planning scans and was interpreted by neuroradiologists. Independent specialist allied health care members assessed patient condition by using morbidity scores including the Waterlow score, ${ }^{22}$ which was completed preoperatively, and a patient handling assessment score and a falls risk assessment score, which were completed postoperatively.

The Waterlow score is a validated pressure sore/ulcer risk assessment tool composed of 7 points: build/weight, continence, skin type/visual risk areas, mobility, sex, age, and appetite. The patient handling score is composed of 3 components: patient build/weight for height, comprehension/behavior-language difficulties, and drowsiness and other factors that might affect handling (e.g., pain severity and whether the patient is receiving an intravenous infusion). The falls risk assessment is an 8-point scoring system that takes into account patient sex, age, sensory deficit, fall history, mobility, medical history, medications, and gait.

Patients who needed radiotherapy or chemotherapy were managed at the Christie Cancer Institute and were followed up at various intervals in the neurooncology outpatient clinic and by telephone consultation. The Christie Cancer Institute electronic patient database was used to identify those patients who had received radiotherapy 
and/or chemotherapy postoperatively, and these parameters were also considered in the analysis. The Christie Hospital is the largest cancer center in Europe; more than 40,000 patients a year receive treatment at this hospital. It was also the first UK center to officially be accredited as a comprehensive cancer center. Patients receive treatment from specialist neurooncology physicians. The standard of treatment at the Christie Hospital is for patients to receive radiotherapy within 1-3 weeks from diagnosis.

\section{Statistical Analyses}

The Statistical Package for the Social Sciences version 17 (SPSS, Inc.) was used to analyze data. Distribution of data was assessed by skewness, kurtosis, and results of the Kolmogorov-Smirnov test. The log-rank test and a proportional hazards model were used to compare survival times for patients in the specialist and generalist groups. Cox regression was used to assess the effects of covariates (type of surgeon, tumor histology [AA or GBM], postoperative receipt of chemotherapy and/or radiotherapy, type of surgery, extent of tumor removal, and case mix) on survival times. The MRC prognostic index was used as an externally validated method to adjust for differences in case mix. Continuous data, including inpatient stay durations and patient morbidity scores, were assessed by using either the Mann-Whitney U-test or the Student t-test, depending on the distribution of patient stay and morbidity scores. The chi-square or Fisher exact test was used to analyze proportional data.

\section{Results}

A total of 306 cases of either GBM or AA were discussed. Of these, 171 were excluded from the study: 89 patients received no intervention because of advanced age, extensive comorbidities, extent of disease, and poor performance status; 74 patients had received chemotherapy or radiotherapy; 5 patients died before treatment; and 3 patients refused treatment. The remaining 135 patients underwent surgery for either GBM $(n=116)$ or AA $(n=19)$; surgery was performed by specialists for 65 patients $(53$ GBM and 12 AA) and by generalists for 70 patients (63 GBM and 7 AA) (Fisher exact test, $p=0.122$ ). Specialists performed 51 craniotomies and 14 stereotactic biopsies; generalists conducted 53 craniotomies and 17 stereotactic biopsies. There was no statistical difference between surgeons in the type of operation performed; however, the numbers of macroscopic resections were higher for specialists than for generalists ( 25 vs 12 , respectively; chi-square $=8.147, \mathrm{p}=0.005$ ) (the spread of confounding variables and outcome variables is illustrated in Table 1) and were a significant predictor of survival (Fig. 1; Cox regression, $\mathrm{p}=0.002$ ).

A crude comparison of survival times demonstrated that median survival time was 290 days (95\% CI 217-363 days) for patients who underwent surgery performed by specialists and 196 days (95\% CI 144-248 days) for those who underwent surgery performed by generalists (logrank test, $p=0.026$ ). Survival times were longer for patients with AA than for those with GBM (log-rank test, $\mathrm{p}$ $=0.001$ ).
Because these results follow a nonrandomized analysis, adjustments for case mix should be considered before interpreting the findings because nonrandomization could add to potential biasing effects. ${ }^{8}$ Because prognosis can be influenced by factors including patient age, history of seizures, tumor pathology, and extent of tumor resection, $, 10,18,19$ we used the MRC brain tumor prognostic index to form a proportional hazards model for the survival data. The index was a highly significant predictor of survival (Cox regression, $\mathrm{p}<0.001$ ); other case mix covariates were assessed for significance.

The effects of case mix were controlled for by using as variables the MRC prognostic index, type of surgeon, extent of tumor resection, tumor pathology, and postoperative treatment with chemotherapy and radiotherapy. Inclusion of these variables demonstrated continued significant improvement in survival times for patients who received treatment from specialists (Cox regression, $\mathrm{p}=0.019$ ) (Fig. 2). There was a trend toward better 30-day mortality rates for patients who received treatment from specialists; only 4 patients (3\%) in the specialist cohort and $10(7 \%)$ in the generalist cohort died. Postoperative chemotherapy and radiotherapy improved survival times (Cox regression, $\mathrm{p}$ $=0.001$ and $\mathrm{p}<0.001$, respectively).

Pressure ulcers developed in 2 patients, 1 in each cohort. Significant differences were not found between the 2 cohorts in terms of Waterlow scores (Mann-Whitney U-test, $\mathrm{p}=0.179$ ), patient handling assessment scores (chisquare $=2.460, p=0.117$ ), or falls risk assessment scores (chi-square $=0.499, \mathrm{p}=0.480$ ). Of note, inpatient stays were shorter among patients who underwent surgery performed by specialists than by generalists (Student $t$-test, $\mathrm{p}$ $=0.008)$ (Fig. 3).

Neuronavigation was used by specialists in 21 operations and by generalists in 19 operations. An independent analysis of neuronavigation use by specialists and generalists demonstrated no added advantage to the extent of resection. Across both groups, neuronavigation was used in 22 of 105 operations, in which it had no influence on the amount of tumor resected (chi-square $=2.911, \mathrm{p}=0.088$ ). Furthermore, its use did not correlate with survival times (log-rank, $\mathrm{p}=0.998)$.

The length of time from referral to multidisciplinary team discussion and from multidisciplinary team discussion to surgery was also analyzed between groups. No difference was found (Mann-Whitney U-test, $\mathrm{p}=0.527$ and $\mathrm{p}$ $=0.373$, respectively).

\section{Discussion}

This study provides evidence in support of subspecialization within surgical neurooncology. At the Greater Manchester Neurosciences Centre, overall survival times were longer for patients who underwent surgery performed by specialist surgical neurooncologists than for those who underwent surgery performed by general neurosurgeons. Furthermore, the 30-day mortality rate was lower for the specialist cohort. Although general neurosurgeons undertook a marginally lower operative caseload, this finding probably just reflects the current trend of referring neurooncology cases to specialists. This trend has enabled a 
TABLE 1. Summary of demographic, treatment, and outcome data

\begin{tabular}{lcclc}
\hline \multicolumn{1}{c}{ Variable } & $\begin{array}{c}\text { Surgery Performed } \\
\text { by Generalist }\end{array}$ & $\begin{array}{c}\text { Surgery Performed } \\
\text { by Specialist }\end{array}$ & Statistical Test & $p$ Value \\
\hline Confounding variable & & & & \\
\hline Tumor histology (GBM/other) & $63 / 7$ & $53 / 12$ & Fisher exact & 0.122 \\
\hline Chemotherapy (yes/no) & $34 / 36$ & $36 / 29$ & Chi-square & 0.268 \\
\hline Radiotherapy (yes/no) & $52 / 18$ & $55 / 10$ & Chi-square & 0.102 \\
\hline Mean performance status score & 51 & 45 & Student t & 0.807 \\
\hline Mean age (yrs) & 57 & 54 & Student t & 0.07 \\
\hline Mean seizure score & 63.1 & 62.8 & Student t & 0.947 \\
\hline Outcome & & & & Log-rank \\
\hline Median survival time (days) & 196 & 290 & Chi-square & 0.026 \\
\hline Extent of resection (total/subtotal) & $12 / 58$ & $25 / 40$ & Student t & 0.005 \\
\hline Mean length of stay (days) & 9 & 5 & Chi-square & 0.008 \\
\hline No. of deaths w/in 30 days & 10 & 4 & 0.122 \\
\hline
\end{tabular}

comparison of the 2 types of surgeons. Our analysis also demonstrated that specialists would more frequently conduct a macroscopic resection, which correlated with improved survival times. The effect of extent of resection on outcome for patients with malignant supratentorial gliomas remains controversial. It has been documented that the extent of GBM tumor resection and residual tumor volume are highly significant factors affecting survival. ${ }^{12,17}$ Retrospective reports have demonstrated that lobectomies are associated with longer survival times. ${ }^{9,11}$ Furthermore, studies primarily evaluating the benefits of postoperative chemotherapy and radiotherapy contain supporting evidence for prolonged survival after total tumor excision..$^{5,21}$ Evidence also exists for a longer time to tumor progression after macroscopic resections, which could also prolong survival times; ${ }^{14}$ imaging studies have confirmed the correlation between lower residual tumor and prolonged survival times. ${ }^{3,23}$

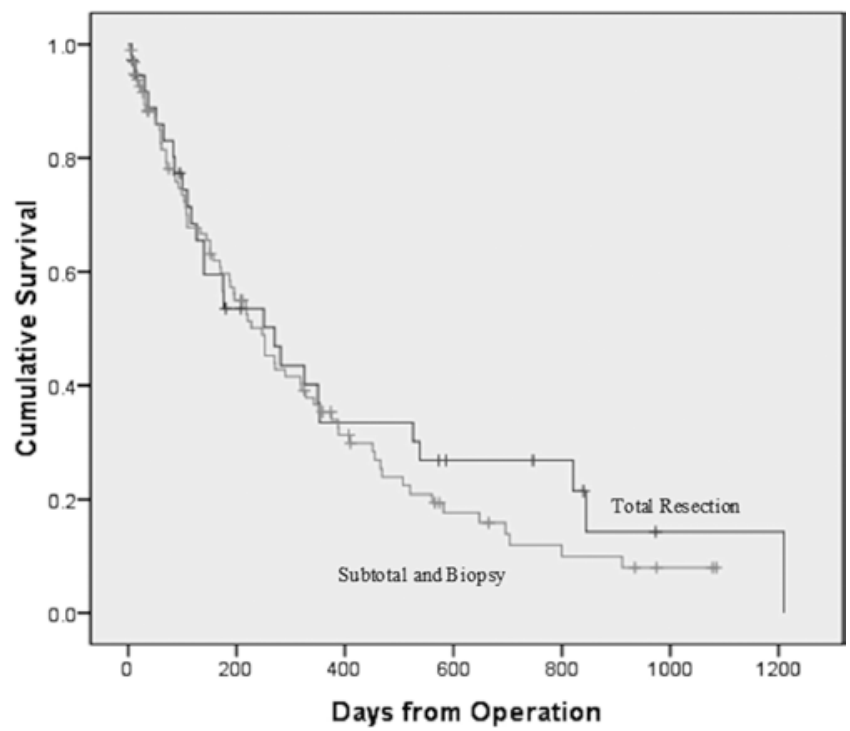

FIG. 1. Kaplan-Meier survival curves comparing survival after total resection of a malignant supratentorial glioma with survival after subtotal resection and biopsy.
Although there has been some concern that extensive resection can lead to greater neurological deficits and morbidity, these outcomes were not experienced by patients in our study and may even favor a reduction in morbidity. ${ }^{2}$ Therefore, therapeutically, our analysis demonstrates a survival advantage after macroscopic tumor resection.

Use of neuronavigation did not statistically influence the extent of tumor resection. This finding might be attributed to the relatively small number of operations in which it was used in our study. Furthermore, the lack of added advantage for completing a macroscopic resection while using neuronavigation might also explain why it did not influence survival times.

The cohort of patients for whom surgery was performed by a specialist also spent significantly less time in hospital after surgery. This finding has many benefits, including reduced cost, less chance of hospital-acquired complications, and possible improvement in patient satisfaction.

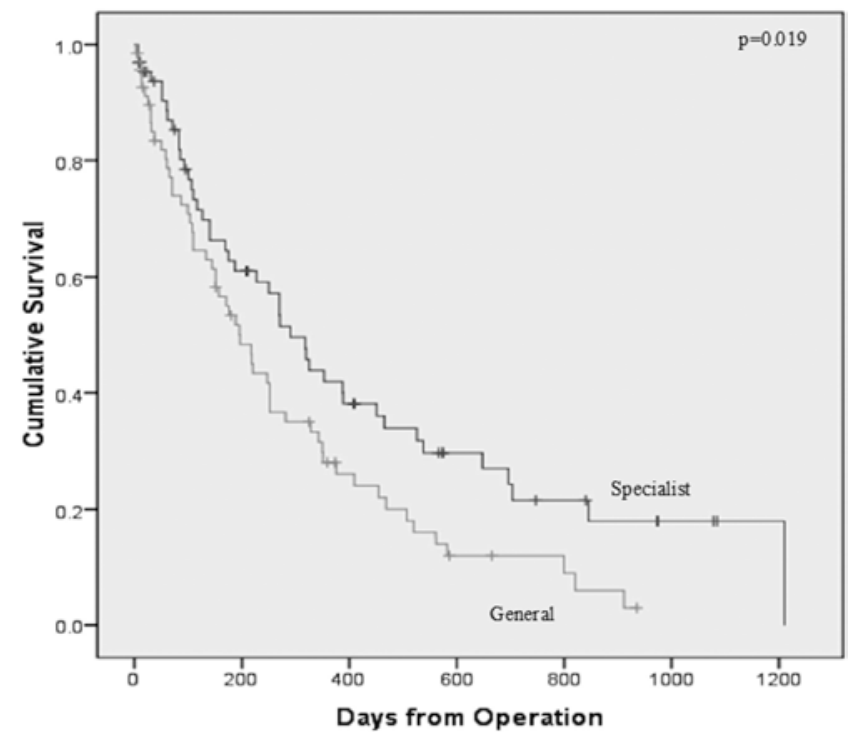

FIG. 2. Kaplan-Meier survival curves showing longer survival after surgical intervention for malignant supratentorial glioma performed by specialist surgical neurooncologists than by general neurosurgeons. 


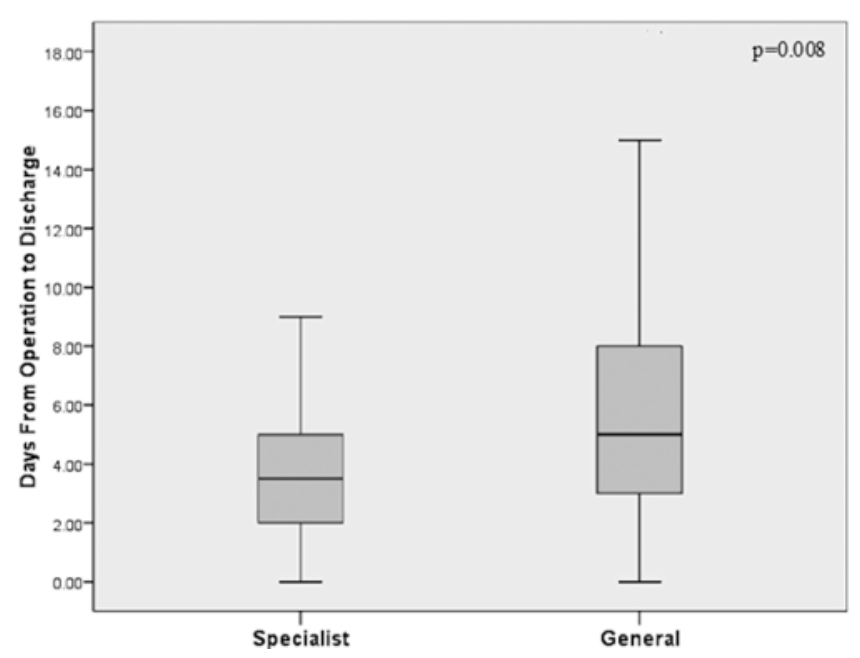

FIG. 3. Box plot with error bars showing shorter inpatient stays after surgical intervention for malignant supratentorial glioma performed by specialist surgical neurooncologists than by general neurosurgeons.

Morbidity scores did not differ; therefore, the diversity in inpatient stay probably did not result from postoperative recovery but rather might depend on the individual surgeon's decision with regard to time of discharge.

Ultimately, if receiving treatment from a specialist neurooncological surgeon provides an outcome advantage, then the reasons are presumably multifactorial. Being a core member of a multidisciplinary neurooncology team facilitates interaction with the whole team caring for patients with brain tumors, including oncologists, radiologists, and specialist nurses. This finding may enable more timely interventions during the many steps in the treatment pathway of patients with CNS tumors.

Our analysis supports the Improving Outcomes Guidance for patients with brain and CNS tumors and provides potential evidence for improved survival times and reduced inpatient stays when surgery is performed by a specialist surgical neurooncologist. These findings may indicate benefits for subspecialization within surgical neurooncology that have yet to be reported.

This study had limitations; however, conducting a more scientific analysis of whether patient outcome is better if treatment is given by a neurooncology specialist neurosurgeon is difficult. If patients were asked whether they would agree to random selection as to whether their treatment would be provided by a specialist or generalist, then recruitment would clearly be challenging. Because our study was retrospective and nonrandomized, it was therefore subject to potential selection bias..$^{15,16}$ However, such bias was limited because we corrected for case mix effects by using a multivariate regression model. In addition, the referral process in our center is unlikely to add to selection bias because there was no case selection; cases were treated by the surgeon on call at the time of referral. An analysis of functional outcome, rather than survival time alone, might also be useful as an outcome measure.

Our findings are from a single-center experience, and given further impetus for subspecialization in many centers, conducting subsequent comparative studies might be difficult. Although most centers in the United Kingdom have a similar setup in terms of cases being discussed in neurooncology multidisciplinary team meetings, patient care is increasingly being taken over by specialists, regardless which surgeon was on call. However, it is important that studies in multiple centers are undertaken with adequate powering to confirm these findings so that in the future, patients with this terminal condition receive the best possible care.

\section{Conclusions}

Our results demonstrate that subspecialization within surgical neurooncology can lead to prolonged patient survival, possibly fewer surgical deaths, and shorter patient hospital stays. Increased amount of tumor resected could contribute to better outcomes. However, our findings remain a single-center experience; to more accurately assess the potential benefits of subspecialization within surgical neurooncology, a multicenter analysis is warranted.

\section{References}

1. Albright AL, Sposto R, Holmes E, Zeltzer PM, Finlay JL, Wisoff JH, et al: Correlation of neurosurgical subspecialization with outcomes in children with malignant brain tumors. Neurosurgery 47:879-887, 2000

2. Ammirati M, Vick N, Liao YL, Ciric I, Mikhael M: Effect of the extent of surgical resection on survival and quality of life in patients with supratentorial glioblastomas and anaplastic astrocytomas. Neurosurgery 21:201-206, 1987

3. Andreou J, George AE, Wise A, de Leon M, Kricheff II, Ransohoff J, et al: CT prognostic criteria of survival after malignant glioma surgery. AJNR Am J Neuroradiol 4:488-490, 1983

4. Burger PC, Vogel FS, Green SB, Strike TA: Glioblastoma multiforme and anaplastic astrocytoma. Pathologic criteria and prognostic implications. Cancer 56:1106-1111, 1985

5. Chang CH, Horton J, Schoenfeld D, Salazer O, Perez-Tamayo $\mathrm{R}$, Kramer S, et al: Comparison of postoperative radiotherapy and combined postoperative radiotherapy and chemotherapy in the multidisciplinary management of malignant gliomas. A joint Radiation Therapy Oncology Group and Eastern Cooperative Oncology Group study. Cancer 52:997-1007, 1983

6. Choudhari KA, Ramachandran MS, McCarron MO, Kaliaperumal C: Aneurysms unsuitable for endovascular intervention: surgical outcome and management challenges over a 5 -year period following International Subarachnoid Haemorrhage Trial (ISAT). Clin Neurol Neurosurg 109:868-875, 2007

7. D'Amelio LF, Hammond JS, Thomasseau J, Sutyak JP: "Adult" trauma surgeons with pediatric commitment: a logical solution to the pediatric trauma manpower problem. Am Surg 61:968-974, 1995

8. Davenport RJ, Dennis MS, Warlow CP: Effect of correcting outcome data for case mix: an example from stroke medicine. BMJ 312:1503-1505, 1996

9. Davis L, Martin J, Goldstein SL, Ashkenazy M: A study of 211 patients with verified glioblastoma multiforme. J Neurosurg 6:33-44, 1949

10. Fine HA: The basis for current treatment recommendations for malignant gliomas. J Neurooncol 20:111-120, 1994

11. Frankel SA, German WJ: Glioblastoma multiforme. Review of 219 cases with regard to natural history, pathology, diagnostic methods, and treatment. J Neurosurg 15:489-503, 1958

12. Keles GE, Anderson B, Berger MS: The effect of extent 
of resection on time to tumor progression and survival in patients with glioblastoma multiforme of the cerebral hemisphere. Surg Neurol 52:371-379, 1999

13. Latif AZ, Signorini DF, Whittle IR: Treatment by a specialist surgical neuro-oncologist does not provide any survival advantage for patients with a malignant glioma. Br J Neurosurg 12:29-32, 1998

14. Levin VA, Hoffman WF, Heilbron DC, Norman D: Prognostic significance of the pretreatment CT scan on time to progression for patients with malignant gliomas. J Neurosurg 52:642-647, 1980

15. Maurice-Williams RS: The notes in the cupboard: the question of intellectual honesty in neurosurgery. Br J Neurosurg 11:277-279, 1997

16. Rothwell P, Warlow C: Is self-audit reliable? Lancet 346:1623, 1995 (Letter)

17. Sanai N, Berger MS: Glioma extent of resection and its impact on patient outcome. Neurosurgery 62:753-766, 2008

18. Shapiro WR, Green SB, Burger PC, Mahaley MS Jr, Selker RG, VanGilder JC, et al: Randomized trial of three chemotherapy regimens and two radiotherapy regimens and two radiotherapy regimens in postoperative treatment of malignant glioma. Brain Tumor Cooperative Group Trial 8001. J Neurosurg 71:1-9, 1989

19. Stenning SP, Freedman LS, Bleehen NM: Prognostic factors for high-grade malignant glioma: development of a prognostic index. A Report of the Medical Research Council Brain Tumour Working Party. J Neurooncol 9:47-55, 1990

20. Stupp R, Mason WP, van den Bent MJ, Weller M, Fisher B, Taphoorn MJB, et al: Radiotherapy plus concomitant and adjuvant temozolomide for glioblastoma. N Engl J Med 352:987-996, 2005

21. Walker MD, Alexander E Jr, Hunt WE, MacCarty CS, Mahaley MS Jr, Mealey J Jr, et al: Evaluation of BCNU and/or radiotherapy in the treatment of anaplastic gliomas. A cooperative clinical trial. J Neurosurg 49:333-343, 1978

22. Waterlow J: Tissue viability. Calculating the risk. Nurs Times 83:58-60, 1987

23. Wood JR, Green SB, Shapiro WR: The prognostic importance of tumor size in malignant gliomas: a computed tomographic scan study by the Brain Tumor Cooperative Group. J Clin Oncol 6:338-343, 1988

\section{Author Contributions}

Conception and design: Khan, Karabatsou, Leggate, Sofat, Kamaly-Asl. Acquisition of data: Khan, Bhavsar. Analysis and interpretation of data: Khan, Bhavsar, Asif. Drafting the article: Khan, Bhavsar, Kamaly-Asl. Critically revising the article: all authors. Reviewed submitted version of manuscript: all authors. Approved the final version of the manuscript on behalf of all authors: Khan. Statistical analysis: Khan. Study supervision: Karabatsou, Leggate, Sofat, Kamaly-Asl.

\section{Supplemental Information} Previous Presentation

Portions of this work were presented in abstract form at the Society of British Neurosurgeons Autumn Meeting, held in London, UK, on September 10, 2010.

\section{Correspondence}

Ursalan Khan, Greater Manchester Neurosciences Centre, Neurosurgery, Salford Royal Hospital, Stott Lane, Salford, Manchester M6 8HD, United Kingdom. email: ursalan@doctors. org.uk. 